\title{
Big Bang Nucleosynthesis Calculation*
}

\author{
Hannu Kurki-Suonio \\ Helsinki Institute of Physics and Department of Physics \\ University of Helsinki, P.O.Box 64, FIN-00014 Helsinki, Finland
}

October 10, 2001

\begin{abstract}
I review standard big bang nucleosynthesis and some versions of nonstandard BBN. The abundances of the primordial isotopes D, He-3, and Li-7 produced in standard $\mathrm{BBN}$ can be calculated as a function of the baryon density with an accuracy of about $10 \%$. For He- 4 the accuracy is better than $1 \%$. The calculated abundances agree fairly well with observations, but the baryon density of the universe cannot be determined with high precision. Possibilities for nonstandard BBN include inhomogeneous and antimatter BBN and nonzero neutrino chemical potentials.
\end{abstract}

\section{Introduction}

Big bang nucleosynthesis (BBN) is among the main observational evidence for big bang. The discovery of the cosmic microwave background (CMB) provided us with the temperature scale of the early universe, and allowed the calculation of the primordial nuclear abundances produced in the big bang. The four light isotopes, $\mathrm{D},{ }^{3} \mathrm{He},{ }^{4} \mathrm{He}$, and ${ }^{7} \mathrm{Li}$ are mainly produced in the big bang, and the calculated abundances agree fairly well with astronomical observations.

Standard big bang nucleosynthesis (SBBN) has a single free parameter, the baryon-to-photon ratio,

$$
\eta \equiv \frac{n_{b}}{n_{\gamma}}=10^{-10} \ldots 10^{-9}
$$

which is related to the present baryonic contribution to the critical density $\Omega_{b}$ via the Hubble constant $H_{0} \equiv h 100 \mathrm{~km} / \mathrm{s} / \mathrm{Mpc}$ by

$$
\eta_{10} \equiv 10^{10} \eta=274 \Omega_{b} h^{2} .
$$

For decades, BBN has provided the best determination of the amount of baryonic matter in the universe. The agreement with observations is obtained in the

\footnotetext{
*Talk given at "Matter in the Universe", ISSI Bern
} 
range $\eta_{10}=1.5 \ldots 6$. Despite optimistic claims from time to time, BBN has not really progressed towards a much more precise determination of $\eta$. Observers claim higher precision from determinations of primordial abundances of single isotopes, but disagree with each other or, within the context of SBBN, with primordial abundances of other isotopes. Difficult questions about systematic errors in observations and chemical evolution relating the present abundances to primordial abundances have prevented further progress.

During the past year, a competing method for estimating the amount of baryonic matter has appeared. In the angular power spectrum of the anisotropy of $\mathrm{CMB}$, the relative heights of the even and odd acoustic peaks are sensitive to the baryon-to-photon ratio. The first preliminary estimates from the Boomerang [29] and Maxima-1 [4] experiments appeared to be in conflict with BBN, giving a higher baryon density, $\Omega_{b} h^{2} \sim 0.03$, or $\eta_{10} \sim 8$ [20]. The Boomerang collaboration has since revised their estimate downward, to $\Omega_{b} h^{2}=0.022_{-0.003}^{+0.004}$ [38], which agrees with SBBN, but the Maxima-1 estimate has been revised upward to $\Omega_{b} h^{2}=0.0325 \pm 0.0125$ (95\% c.l.) [53. With the coming satellite experiments CMB may surpass BBN as the method for estimating $\eta$. BBN will then become a tool for understanding the astrophysics of chemical evolution, by telling us the primordial abundances.

While SBBN is simple and natural, and is at present in reasonable agreement with observations, there is interest in studying nonstandard variants of BBN. For one thing, BBN is a sensitive probe of the physics of the early universe. If we change something about our assumptions regarding the conditions in the early universe, or the physics relevant at that time, we are likely to change the primordial abundances and ruin the agreement with observations. Thus for many things BBN provides the strongest constraint.

On the other hand, from time to time there have been suggestions for disagreement between the estimated primordial abundances of the different isotopes, and/or other ways of estimating $\eta$. If such disagreements persist, nonstandard BBN (NSBBN) may be the solution.

I shall begin with a review of the physics of SBBN, and then discuss a few NSBBN scenarios.

\section{Physics of Big Bang Nucleosynthesis}

In the early universe the temperature is falling as the universe expands. The time scale depends on the number of particle species which are relativistic at that time. In the standard case these are electrons, positrons, photons, and 3 species of neutrinos.

There is lots of radiation and very little matter. The amount of baryonic matter is not known exactly, and is given by $\eta$, the only free parameter in SBBN.

Weak reactions are converting neutrons into protons. At first the reaction rate is high enough to maintain the equilibrium neutron-to-proton ratio

$$
\left(\frac{n}{p}\right)_{\mathrm{eq}}=e^{-\left(m_{n}-m_{p}\right) / T-\xi_{e}},
$$


which is falling with temperature. Here $\xi_{e} \equiv \mu_{\nu_{e}} / T$ is the electron neutrino degeneracy parameter $\left(\mu_{\nu_{e}}\right.$ is the electron neutrino chemical potential). For $\xi \ll 1$, we have roughly

$$
\xi \sim \frac{n_{\nu}-n_{\bar{\nu}}}{n_{\gamma}}
$$

In SBBN we assume homogeneous conditions with $\xi_{e} \sim 0$.

At a temperature of about $T \sim 0.8 \mathrm{MeV}$ the neutrinos decouple and after that the neutron abundance evolves via free neutron decay

$$
n \rightarrow p+e^{-}+\bar{\nu}_{e} .
$$

Nuclear reactions begin by neutrons and protons producing deuterium. This reaction keeps the deuterium abundance close to its equilibrium value. Because of the large amount of photons in the background radiation and the low binding energy, $B_{d}=2.22 \mathrm{MeV}$, of deuterium, the deuterium abundance does not become large until the temperature has fallen to about $70 \mathrm{keV}$. Only then can the nuclear reactions proceed beyond deuterium.

As the temperature falls further the Coulomb barrier shuts down the nuclear reactions. Because of the short time and bottlenecks due to the lack of stable nuclei at masses $A=5$ and $A=8$, the reactions do not proceed beyond $A=7$.

There are 10 important reactions which take nucleosynthesis beyond deuterium:

$$
\begin{array}{llll}
d(p, \gamma)^{3} \mathrm{He} & d(d, p) t & d(d, n)^{3} \mathrm{He} & { }^{3} \mathrm{He}(n, p) t \\
t(d, n)^{4} \mathrm{He} & { }^{3} \mathrm{He}(d, p){ }^{4} \mathrm{He} & { }^{3} \mathrm{He}(\alpha, \gamma)^{7} \mathrm{Be} & t(\alpha, \gamma){ }^{7} \mathrm{Li} \\
{ }^{7} \mathrm{Be}(n, p)^{7} \mathrm{Li} & { }^{7} \mathrm{Li}(p, \alpha)^{4} \mathrm{He} & &
\end{array}
$$

Afterwards tritium $\beta$-decays into ${ }^{3} \mathrm{He}$ and ${ }^{7} \mathrm{Be}$ becomes ${ }^{7} \mathrm{Li}$ by electron capture.

Since ${ }^{4} \mathrm{He}$ has the highest binding energy per nucleon (for $A \leq 7$ ), almost all neutrons end up incorporated in ${ }^{4} \mathrm{He}$. Thus the primordial ${ }^{4} \mathrm{He}$ abundance $Y_{p}$ is determined by the neutron fraction at nucleosynthesis time. This in turn is determined by the competition between the weak reaction rates and the rate at which the temperature falls due to the expansion of the universe. A higher baryon density causes nucleosynthesis to take place at a higher temperature, when there are more neutrons left. Thus $Y_{p}$ increases with $\eta$.

A higher $\eta$ means more efficient nuclear burning to ${ }^{4} \mathrm{He}$, leaving less of the "impurities", D and ${ }^{3} \mathrm{He}$. There is also less directly produced ${ }^{7} \mathrm{Li}$, but a higher density allows more ${ }^{7} \mathrm{Be}$ to be produced, so that the final primordial ${ }^{7} \mathrm{Li}$ abundance has a more complicated dependence on $\eta$ (see figure 1).

\section{Accuracy of Nucleosynthesis Yields}

The accuracy of the SBBN calculation of produced primordial abundances depends on how accurately the various thermonuclear reaction rates are known. These rates cannot be calculated from first principles at present, and are thus based on laboratory measurements. BBN codes make use of compilations of 
these rates [13, 14, 18, 77, 8]. The Caughlan\&Fowler [8] 1988 compilation was updated on some key BBN rates by Smith et al. [51]. The latest compilation is by the NACRE collaboration [2].

For BBN, the most significant difference between the new NACRE rates and the older rates is that deuterium production is now higher. With the NACRE rates, the O'Meara et al. [39] result $\mathrm{D} / \mathrm{H}=3.0 \pm 0.4 \times 10^{-5}$ corresponds to $\eta_{10}=5.9 \pm 0.5$ instead of $\eta_{10}=5.6 \pm 0.5$ obtained with the older rates.

Based on how accurately the reaction rates are known, Cyburt et al. [9] have estimated the accuracy of SBBN yields in the range $\eta_{10}=1 \ldots 10$ to be better than $0.3 \%$ for $Y_{p}, 3-7 \%$ for $\mathrm{D}, 3-10 \%$ for ${ }^{3} \mathrm{He}$, and $12-19 \%$ for ${ }^{7} \mathrm{Li}$.

\section{Observations}

I shall leave the more detailed discussion of the observations relating to primordial abundances to other participants of this workshop, and just list various recent results in the literature.

For ${ }^{4} \mathrm{He}$, Olive et al. 40 combined results of different observers and estimated a primordial abundance $Y_{p}=0.234 \pm 0.003$. Izotov\&Thuan 19 used their own large set of observations to arrive at $Y_{p}=0.244 \pm 0.002$. The errors are 1- $\sigma$ statistical errors. There has been much recent discussion of possible systematic errors. Peimbert et al. [41] have recently measured the ${ }^{4} \mathrm{He}$ abundance in the SMC, whose proximity allows a better control of systematic errors. They obtained $Y=0.2405 \pm 0.0018$ for the SMC, and from this they estimate $Y_{p}=0.2345 \pm 0.0026$ for the primordial abundance. Thuan\&Izotov 55 have now refined their estimate to $Y_{p}=0.2443 \pm 0.0015$. After discussing various systematic effects, they conclude that because of systematic errors this could be an underestimate by $\sim 2-4 \%$.

The observed deuterium abundance in the local interstellar medium is $\mathrm{D} / \mathrm{H}=$ $1.50 \pm 0.10 \times 10^{-5}$ [33], but there is evidence for local variations [57]. For example, Sonneborn et al. 52 have recently reported $\mathrm{D} / \mathrm{H}=2.18_{-0.31}^{+0.36} \times 10^{-5}$ along one line of sight. Since deuterium is destroyed in stellar processes, its primordial abundance should be greater than the local abundance.

The most promising method of obtaining the primordial deuterium abundance is the measurement of $\mathrm{D} / \mathrm{H}$ from Lyman-series absorption by high-redshift clouds. Based on three such measurements and upper limits from others, O'Meara et al. 39] obtain $\mathrm{D} / \mathrm{H}=3.0 \pm 0.4 \times 10^{-5}$. Measurements on one such system suggest a higher deuterium abundance, possibly larger than $10^{-4}$ [58, 56]. Recently, the deuterium in two more such systems has been observed [10, 32, 42], supporting the low $\mathrm{D} / \mathrm{H}$ of O'Meara et al. 39].

The estimates for the primordial ${ }^{7} \mathrm{Li}$ abundance are based on the rather uniform abundances in population II halo stars. Bonifacio\&Molaro [5] obtained $\log _{10}\left({ }^{7} \mathrm{Li} / \mathrm{H}\right)=-9.80 \pm 0.012 \pm 0.05$ and Ryan et al. 47 obtained $\log _{10}\left({ }^{7} \mathrm{Li} / \mathrm{H}\right)=-9.88$ for the mean abundance. This measured abundance is close to the minimum ${ }^{7} \mathrm{Li}$ from SBBN (see figure 1). It may be depleted somewhat from the primordial abundance by stellar processing. Pinsonneault et 

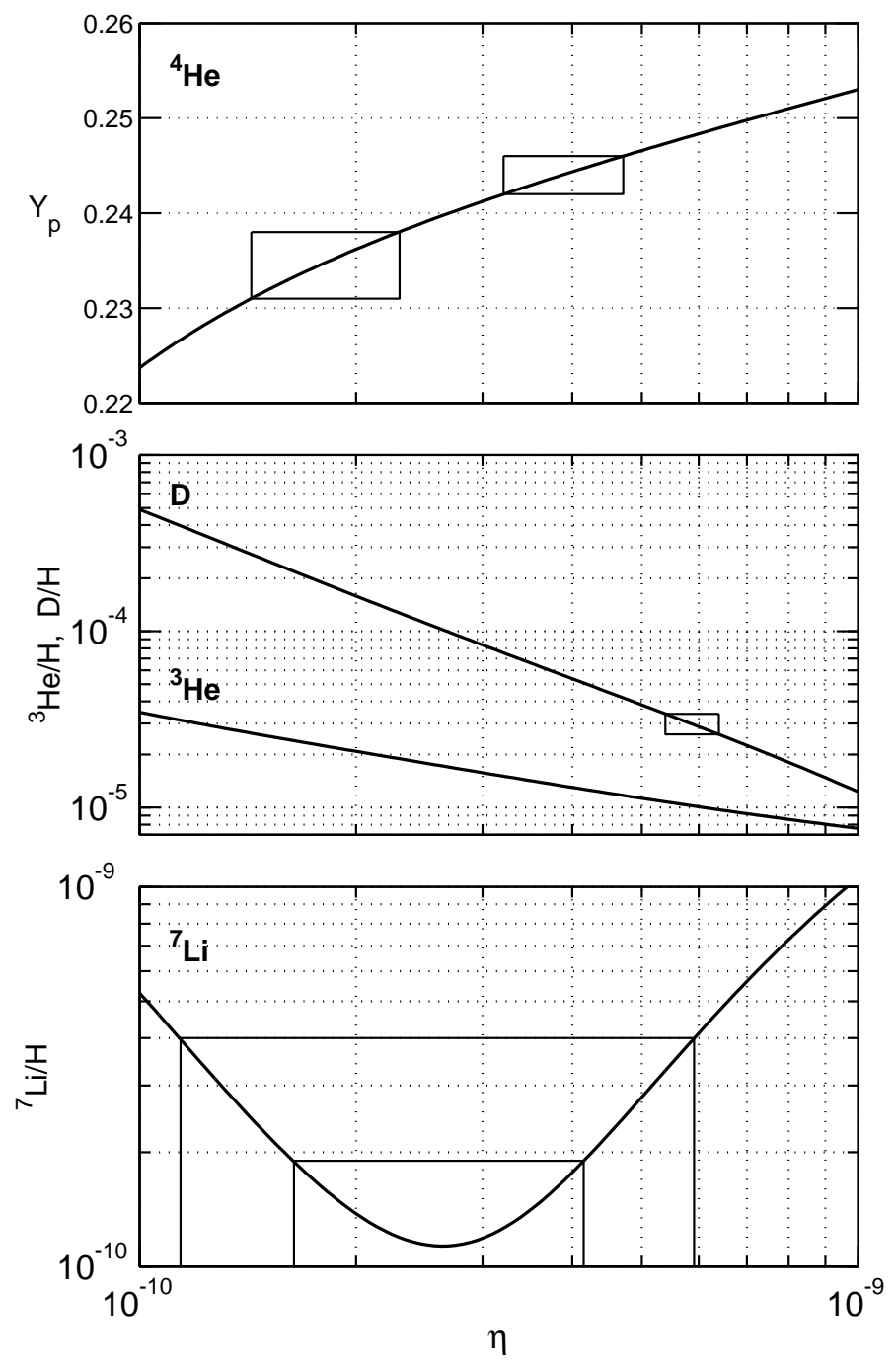

Figure 1: The SBBN yields of the light isotopes as a function of the baryonto-photon ratio $\eta$. The rectangles corresponds to the various observational estimates for the primordial abundances mentioned in the text: ${ }^{4} \mathrm{He}$ is from Olive et al. 40] and Izotov\&Thuan [19], $\mathrm{D}$ is from O'Meara et al. [39] and ${ }^{7} \mathrm{Li}$ is from Ryan et al. 48]. For ${ }^{7} \mathrm{Li}$ a conservative upper $\operatorname{limit} \log _{10}\left({ }^{7} \mathrm{Li} / \mathrm{H}\right) \leq-9.4$ is also shown. Figure from [50]. 
al. 433] estimate $\log _{10}\left({ }^{7} \mathrm{Li} / \mathrm{H}\right)=-9.65 \ldots-9.25$ for the primordial abundance. The observed ${ }^{7} \mathrm{Li}$ may also include a galactic contribution, so that the primordial abundance could be even lower. For the primordial abundance Ryan et al. [48] estimate $\log _{10}\left({ }^{7} \mathrm{Li} / \mathrm{H}\right)=-10.04 \ldots-9.72$ and Suzuki et al. [54] estimate $\log _{10}\left({ }^{7} \mathrm{Li} / \mathrm{H}\right)=-9.97 \ldots-9.77$.

Comparing these estimates for primordial abundances to the SBBN yields (figure 1) we see that there is some tension between $\mathrm{D} / \mathrm{H}$, which favors a higher baryon density, and $Y_{p}$ and ${ }^{7} \mathrm{Li} / \mathrm{H}$, which favor a lower baryon density. The best estimate from CMB now agrees with the high $\eta$ from D/H. Thuan\&Izotov [55] now conclude that their value for $Y_{p}$ is in good agreement with this when one allows for the systematic error. If one accepts the Thuan\&Izotov [55] result for $Y_{p}$ the remaining disagreement is with the ${ }^{7} \mathrm{Li}$. Possibly ${ }^{7} \mathrm{Li}$ processing is not yet understood well enough, and the tighter limits above are too stringent.

\section{Nonstandard BBN}

There are many proposed possible modifications to SBBN. I shall go over just a few of these NSBBN scenarios.

A higher energy density at a given temperature would lead to faster expansion in the early universe. This would be caused by the presence of additional relativistic particle species, e.g., additional neutrino species. The shorter time scale would mean that there are more neutrons left at nucleosynthesis time, and thus more ${ }^{4} \mathrm{He}$ is produced. This is the most important effect. Since a higher $Y_{p}$ tends to lead to worse agreement with observations, BBN sets an upper limit to the speed-up possible. This upper limit is usually parameterized in terms of the effective number of (light) neutrino species $N_{\nu}$. Different authors get different upper limits depending on the observational constraints chosen. Two recent results are $N_{\nu}<3.2$ by Burles et al. [6] and $N_{\nu} \leq 4$ by Lisi et al. [34].

The effect on other isotopes is roughly that their abundance curves vs. the baryon density are shifted towards higher $\eta$. The higher density compensates for the shorter time available. This is a smaller effect than the effect on ${ }^{4} \mathrm{He}$.

A large neutrino degeneracy, $\xi \neq 0$, would increase the energy density in neutrinos at a given temperature, also leading to a speed-up of the expansion rate. If the degeneracy is in electron neutrinos, a much larger effect is that $\xi_{e} \neq 0$ shifts the balance of weak reactions (see eq. 3). If $\xi_{e}>0$, so that there are more electron neutrinos than electron antineutrinos, we get less neutrons and thus a lower $Y_{p}$. Since this has a large effect on $Y_{p}$ but a small effect on the other isotopes, we can use $\xi_{e}$ to "dial in" a desired value of $Y_{p}$, as $\xi_{e}$ is otherwise unobservable.

One can combine the above scenarios by having both a significantly faster expansion rate and $\xi_{e}>0$, so that the effects on $Y_{p}$ cancel each other to keep it in the observational range, while the effect of the speed-up on the other isotopes remains [23]. If one wants to use neutrino degeneracy only to achieve this speedup, this scenario requires $\left|\xi_{\mu}\right| \gg \xi_{e}$ or $\left|\xi_{\tau}\right| \gg \xi_{e}$. This way larger $\eta$ are allowed than in SBBN. However, since one is relying on a small effect, the large effects 


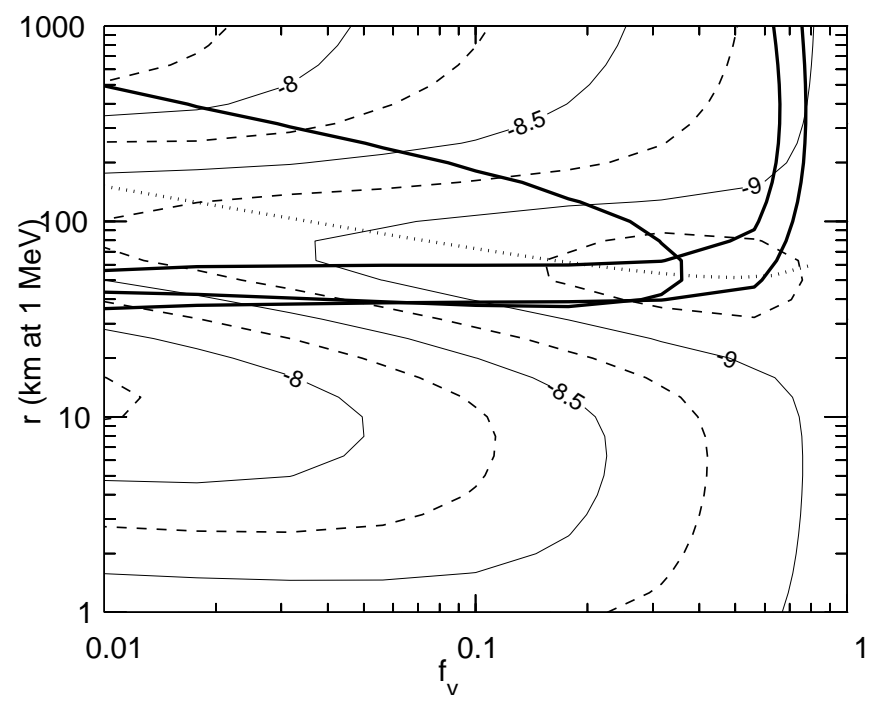

Figure 2: Results from IBBN for $\Omega_{b} h^{2}=0.030\left(\eta_{10}=8.22\right)$. This is the density suggested by the preliminary Boomerang and Maxima-1 results. $r$ is the distance scale giving the separation between centers of high- and low-density regions, and $f_{v}$ is the volume fraction of the high-density regions. The distance scale is given in comoving units at $T=1 \mathrm{MeV}$. $\left(1 \mathrm{~km}\right.$ at $1 \mathrm{MeV}$ corresponds to $1.9 \times 10^{-4} \mathrm{pc}$ at present.) The thick lines show the observational constraints $Y_{p} \leq 0.248$ (to the left of the curve) and $\mathrm{D} / \mathrm{H}=2.9-4.0 \times 10^{-5}$. IBBN can thus bring this high $\eta$ into agreement with ${ }^{4} \mathrm{He}$ and $\mathrm{D}$ observations. Thin lines are contours of $\log _{10}\left({ }^{7} \mathrm{Li} / \mathrm{H}\right)$, showing that ${ }^{7} \mathrm{Li}$ remains problematically high. This figure is for a "spherical shell" geometry which is more successful in reducing the ${ }^{7} \mathrm{Li}$ yield than other geometries tried. The dotted curve shows where figure 3 cuts through this figure. Figure from 28].

having cancelled each other, this scenario requires a very large speed-up for a significant effect. A large $N_{\nu}$ leads to other cosmological effects which put limits to this scenario. In particular, the recent CMB anisotropy results place an upper bound to $N_{\nu}$ [15, 16]. When the CMB constraints are combined with this $\mathrm{BBN}$ scenario [12, 24, 31, 17] the conclusion is that the upper limit to $\eta_{10}$ can be raised from the SBBN $\eta_{10}<6$ to $\eta_{10} \leq 7 \ldots 8$ only [24].

\section{Inhomogeneous BBN}

Another way to modify SBBN is to relax the homogeneity assumption, and allow $\eta$ to be a function of position at nucleosynthesis time. In the usual kind of inhomogeneous BBN (IBBN), the baryon density is positive, $\eta>0$, everywhere, but one can also consider the possibility of having antimatter regions, where $\eta<0$. This latter case I call antimatter BBN (ABBN), and I discuss it in the 


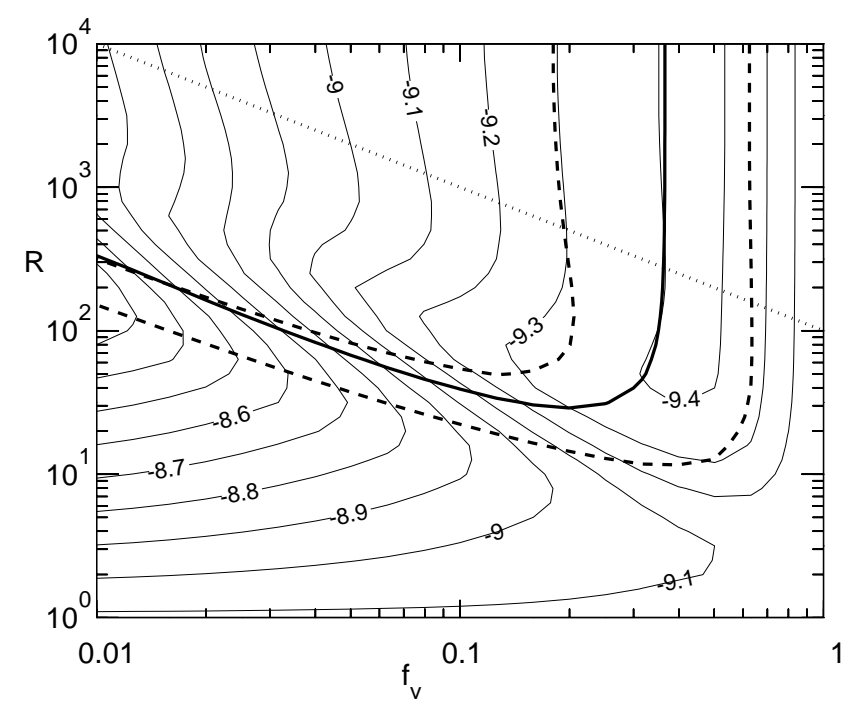

Figure 3: Like figure 2, but now we show the dependence on the third IBBN parameter, the density contrast $R$ between the high- and low-density regions. The dotted line shows where figure 2 cuts through this one. The solid thick line is the constraint $Y_{p} \leq 0.248$ (above the line) and the dashed thick lines give the $\mathrm{D} / \mathrm{H}$ constraint. The thin lines are the $\log _{10}\left({ }^{7} \mathrm{Li} / \mathrm{H}\right)$ contours. This model can just reach below our "conservative upper limit" $\log _{10}\left({ }^{7} \mathrm{Li} / \mathrm{H}\right) \leq-9.4$. Figure from 28$]$. 
next section.

The crucial question in IBBN is the distance scale $r$ of the inhomogeneity. If the mechanism causing the inhomogeneity is connected with inflation, inhomogeneity at any scale could be produced. The observed isotropy of the CMB, however, requires $\eta$ to be highly homogeneous at distance scales larger than about $10 \mathrm{Mpc}$. Thus IBBN cannot be used to explain, e.g., different deuterium abundances at different high-redshift Lyman-absorbers.

In the usual IBBN scenarios one assumes a much smaller distance scale of the inhomogeneity, so that while $\eta$ is inhomogeneous at nucleosynthesis time, the matter from different regions gets mixed later, resulting in a homogeneous baryon density with homogeneous abundances, which however are different from the SBBN abundances for the same $\eta$.

In the simplest version of IBBN, each small region undergoes SBBN with its own baryon-to-photon ratio, and one gets the final abundances by averaging over the SBBN abundances over the distribution in $\eta$. Leonard\&Scherrer [30] have shown that this way one can relax, or even remove, the observational lower limit to $\eta$, but the upper limit to $\eta$ remains unchanged.

If the mechanism producing the inhomogeneity was not connected with inflation, causality requires the distance scale to be smaller than the horizon at that time. A favorite candidate for producing the inhomogeneity has been the QCD phase transition at $T \sim 150 \mathrm{MeV}$, when the horizon was about $1 \mathrm{pc}$ (comoving). The order of the phase transition is not known, but if it was of first order, it would have proceeded through nucleation of bubbles of hadronic matter in the ambient quark-gluon plasma. Near the end of the transition there would have been shrinking droplets of quark-gluon plasma, where much of the baryon number would have been concentrated due to the difficulty of baryon number crossing the phase boundary. This way very high density contrasts could be produced. The relevant distance scale is close to the neutron diffusion scale at nucleosynthesis time. An inhomogeneity of this scale would produce a strongly inhomogeneous neutron-to-proton ratio due to neutron diffusion out of the highdensity regions, leading to a drastic change in $\mathrm{BBN}$ [3, 1, 35]. In the first papers the effect was overestimated; for more accurate calculations see, e.g., [25, 37, 21]. A more extensive list of references is given in the review articles by Reeves [44] and Malaney\&Mathews [36] and in, e.g., 222]. For a range of distance scales one can get less ${ }^{4} \mathrm{He}$ and more D than in SBBN, favoring a higher $\eta$. One can also get some reduction in the ${ }^{7} \mathrm{Li}$ yield, but not much. Because of the large number of parameters in the scenario, quantitative results are not easily summarized. The IBBN scenario is contrasted with recent observations in 22] and 28].

The properties of the QCD phase transition can be estimated by lattice QCD calculations. While a distance scale interesting for IBBN can not be ruled out, unfortunately a too small distance scale appears more likely. 


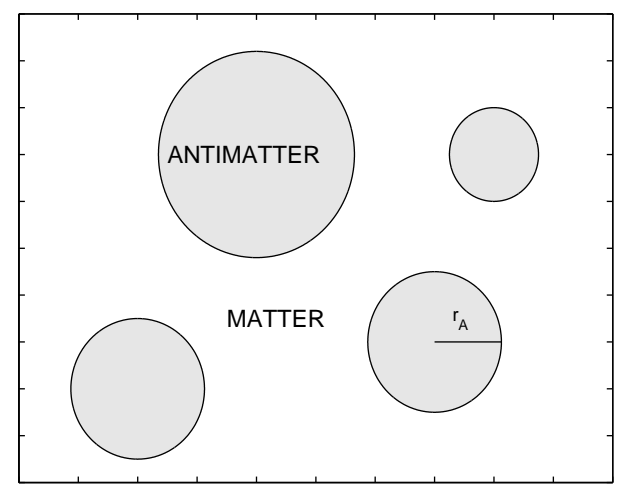

Figure 4: Antimatter regions. Figure from 50.

\section{$7 \quad$ BBN with Antimatter Regions}

We do not know the origin of the baryoasymmetry, $\eta>0$, of the universe. Theories of baryogenesis try to explain this excess of matter over antimatter and to predict (postdict) the observed value of $\eta$. While the simplest models usually lead to a homogeneous $\eta$, in many baryogenesis models the baryon density may come out inhomogeneous, and in some regions the asymmetry may have the opposite sign, so that after local annihilation we end up with regions of antimatter [11].

Annihilation will then proceed at the matter-antimatter boundary. The smaller the antimatter regions the sooner they are completely annihilated. From the spectrum of the CMB we know that there was no major annihilation going on close to recombination time, and the observed cosmic diffuse gamma ray radiation puts tight limits on annihilation after recombination. This leaves us three possibilities not in contradiction with observations: 1) the antimatter regions annihilated well before recombination time, 2) the amount of antimatter was much less (a factor of about $10^{-6}$ or less) than the amount of matter, or 3 ) the antimatter regions are separated by large distances, comparable to the present horizon, or larger.

The first possibility leads to an interesting NSBBN scenario, antimatter $\mathrm{BBN}(\mathrm{ABBN})$ 44, 45, 46, 26, 26, 27, 49. We consider antimatter regions which are larger than $10^{-5} \mathrm{pc}$ but smaller than $100 \mathrm{pc}$ (comoving). These annihilate after $T=1 \mathrm{MeV}$, but before recombination, and the annihilation process can significantly affect nucleosynthesis, or modify the abundances afterwards. Since in the end we have complete annihilation of the antimatter, we need to assume an excess of matter over antimatter, so that the antimatter/matter ratio $R<1$.

Antimatter regions smaller than $10^{-2.5} \mathrm{pc}$ annihilate before nucleosynthesis, when the temperature of the universe is $T=1 \mathrm{MeV}-70 \mathrm{keV}$. The mixing of matter and antimatter is due to (anti)neutron diffusion, and therefore the annihilation is preferably on neutrons. This reduces the ${ }^{4} \mathrm{He}$ production. The 


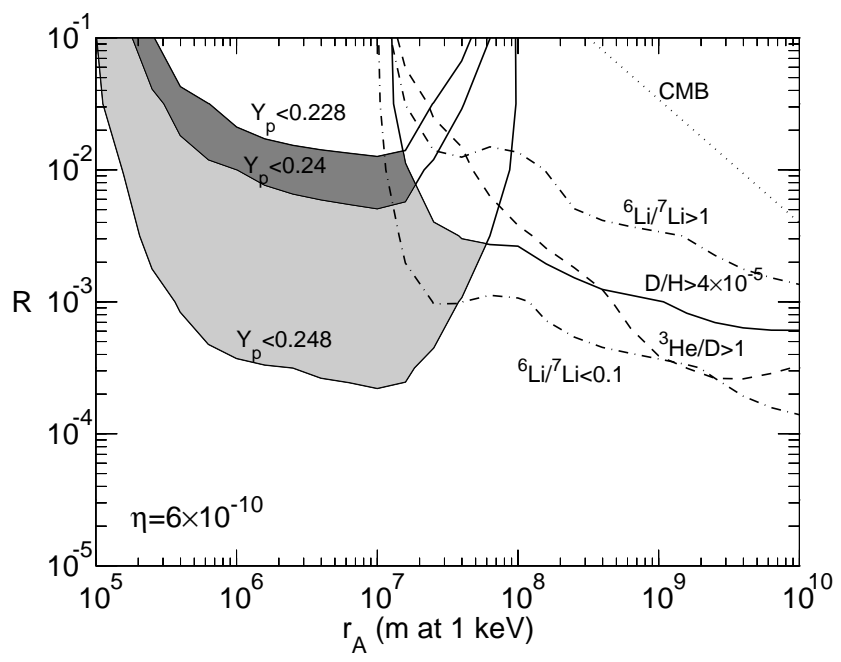

Figure 5: Observational constraints for $\mathrm{ABBN}$ with $\eta_{10}=6 . R$ is the antimatter/matter ratio, and $r_{A}$ is the radius of the antimatter region, given in comoving units at $T=1 \mathrm{keV}$. ( $1 \mathrm{~m}$ at $1 \mathrm{keV}$ is $4.26 \times 10^{6} \mathrm{~m}=1.38 \times 10^{-10} \mathrm{pc}$ today.) The shaded region is the region allowed by the constraints $Y_{p}=0.228-0.248$ and $\mathrm{D} / \mathrm{H}=2.2-4.0 \times 10^{-5}$. $\mathrm{ABBN}$ is thus able to remove the tension between $\mathrm{D}$ and ${ }^{4} \mathrm{He}$ observations. This region is also allowed by other constraints: the spectral distortion of the $\mathrm{CMB}$ and upper limits to ${ }^{3} \mathrm{He} / \mathrm{D}$; the excluded regions are above and to the right of these curves. Because ${ }^{6} \mathrm{Li}$ is very fragile, it is difficult to make definitive conclusions about its primordial abundance from observations. Therefore we just show two contours, ${ }^{6} \mathrm{Li} /{ }^{7} \mathrm{Li}=0.1$ and ${ }^{6} \mathrm{Li} /{ }^{7} \mathrm{Li}=1$. The ${ }^{7} \mathrm{Li}$ yield is fairly constant, close to the $\mathrm{SBBN}$ value ${ }^{7} \mathrm{Li} / \mathrm{H}=2.8 \times 10^{-10}$, over the whole figure. Figure from [49].

observational lower limit to $Y_{p}$ therefore provides an upper limit to the amount of antimatter $R$.

Larger antimatter regions survive until nucleosynthesis, which consumes the free neutrons. This stops further annihilation for a while. There is then a second burst of annihilation well after nucleosynthesis, when proton diffusion becomes effective, at $T<3 \mathrm{keV}$. The annihilation process then changes the nuclear abundances through several mechanisms.

Gamma rays from annihilation photodisintegrate nuclei. The major effect is the production of D and especially ${ }^{3} \mathrm{He}$ from photodisintegration of ${ }^{4} \mathrm{He}$.

Antiproton annihilation on helium nuclei produces ${ }^{3} \mathrm{H},{ }^{3} \mathrm{He}, \mathrm{D}$, protons and neutrons. These nuclear remnants have high energies, and the energetic ${ }^{3} \mathrm{H}$ lead to nonthermal production of ${ }^{6} \mathrm{Li}$ by the endoergic reaction $t(\alpha, n)^{6} \mathrm{Li}$.

Thus $\mathrm{D},{ }^{3} \mathrm{He}$, and ${ }^{6} \mathrm{Li}$ abundances are increased over the SBBN yields. There is no big effect on ${ }^{7} \mathrm{Li}$. 


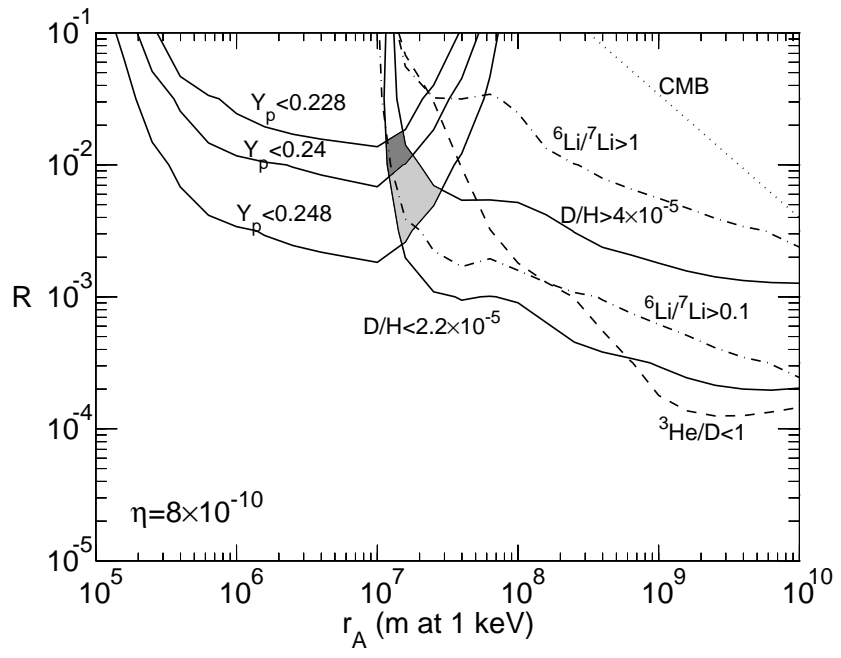

Figure 6: Same as the previous figure, but with $\eta_{10}=8$. ABBN can accommodate higher values of $\eta$ than SBBN, except for the ${ }^{7} \mathrm{Li}$ constraint, which is essentially the same for ABBN and SBBN. Figure from [49.

At $T<1 \mathrm{keV}$, the energy released in annihilation does not get fully thermalized, but results in a distortion of the CMB spectrum. Since no such distortion has been observed, CMB places constraints an the amount of antimatter annihilating below $T \sim 1 \mathrm{keV}$, but before recombination. Near $T \sim 1 \mathrm{keV}$ the universe is still strongly radiation dominated, so the energy release in annihilation is small compared to the energy in the background radiation. As the temperature falls, the matter-to-radiation energy ratio increases. Therefore the limits from CMB to the amount of antimatter become progressively stronger for larger distance scales, for which the annihilation occurs later. For scales smaller than $1 \mathrm{pc}$, we get, however, a stronger limit from ${ }^{3} \mathrm{He}$ overproduction.

Since ABBN reduces $Y_{p}$ and increases $\mathrm{D} / \mathrm{H}$, it allows for a larger baryon density, at least from those observational constraints. The constraint from ${ }^{7} \mathrm{Li}$, however, remains essentially unchanged.

\section{Conclusions}

Standard BBN is compelling in its simplicity. While there is controversy among the observers and some apparent discrepancy between the estimated primordial abundances of the different isotopes and SBBN, these are probably not serious, and most likely represent difficulties in making observations and estimating primordial abundances based on observed ones. SBBN is thus not in trouble. Unfortunately, because of these difficulties, it is not able to pin down the baryonto-photon ratio very precisely. It is somewhere in the range $\eta_{10}=1.5-6$, or 
$\Omega_{b} h^{2}=0.005-0.022$. The high redshift deuterium measurements point towards the upper end of this range, $\eta_{10} \sim 5-6$, or $\Omega_{b} h^{2} \sim 0.02$.

The recent estimates from CMB anisotropy, $\Omega_{b} h^{2}=0.022_{-0.003}^{+0.004}$ from Boomerang [38] and $\Omega_{b} h^{2}=0.0325 \pm 0.0125$ (95\% c.l.) from Maxima-1 [53] also favor this upper end of the range. We are eagerly waiting for more precise CMB measurements in the coming years.

While standard $\mathrm{BBN}$ is in good shape, there is interest in studying nonstandard BBN: to assess the robustness of SBBN, to constrain possibilities for nonstandard physics or cosmology, and to be ready to provide relief if observational discrepancies turn out to be serious for SBBN.

We discussed here four NSBBN scenarios: 1) electron neutrino degeneracy, 2) electron neutrino degeneracy combined with a speed-up of the expansion rate due to extra energy density, 3) inhomogeneous BBN, and 4) antimatter BBN. All these scenarios are able to relieve the tension between the D and ${ }^{4} \mathrm{He}$ observations. The three last ones may also allow a larger baryon density than SBBN, but with some difficulty: 2) is constrained in that respect by other cosmological constraints and 3) and 4) cannot do much for the ${ }^{7} \mathrm{Li}$ constraint.

I thank Elina Sihvola for permission to reproduce figures from [49, 50].

\section{References}

[1] Alcock, C., Fuller, G.M. and Mathews, G.J.: 1987, Astrophys. J. 320, 439.

[2] Angulo, C. et al. (NACRE Collaboration): 1999, Nucl. Phys. A656, 3.

[3] Applegate, J.H., Hogan, C.J. and Scherrer, R.J.: 1987, Phys. Rev. D 35, 1151.

[4] Balbi, A. et al.: 2001, Astrophys. J. 545, L1.

[5] Bonifacio, P. and Molaro, P.: 1997, Mon. Not. R. Astron. Soc. 285, 847.

[6] Burles, S., Nollett, K.M., Truran, J.W. and Turner, M.S.: 1999, Phys. Rev. Lett. 82, 4176.

[7] Caughlan, G.R., Fowler, W.A., Harris, M.J and Zimmerman, B.A.: 1985, At. Data Nucl. Data Tables 32, 197.

[8] Caughlan, G.R. and Fowler, W.A.: 1988, At. Data Nucl. Data Tables 40, 283.

[9] Cyburt, R.H., Fields, R.D., Olive, K.A.: 2001, astro-ph/0102179.

[10] D'Odorico, S., Dessauges-Zavadsky, M. and Molaro, P.: 2001, astro$\mathrm{ph} / 0102162$.

[11] Dolgov, A.D.: 1996, hep-ph/9605280. 
[12] Esposito, S., Mangano, G., Melchiorri, A., Miele, G. and Pisanti, O.: 2001, Phys. Rev. D 63, 043004.

[13] Fowler, W.A., Caughlan, G.R. and Zimmerman, B.A.: 1967, Ann. Rev. Astron. Ap. 5, 525.

[14] Fowler, W.A., Caughlan, G.R. and Zimmerman, B.A.: 1975, Ann. Rev. Astron. Ap. 13, 69.

[15] Hannestad, S.: 2000, Phys. Rev. Lett. 85, 4203.

[16] Hannestad, S.: 2001, Phys. Rev. D 64, 083002.

[17] Hansen, S.H., Mangano, G., Melchiorri, A., Miele, G. and Pisanti, O.: 2001, astro-ph/0105385.

[18] Harris, M.J., Fowler, W.A., Caughlan, G.R. and Zimmerman, B.A.: 1983, Ann. Rev. Astron. Ap. 21, 165.

[19] Izotov, Y.I. and Thuan, T.X.: 1998, Astrophys. J. 500, 188.

[20] Jaffe, A.H. et al.: 2001, Phys. Rev. Lett. 86, 3475.

[21] Jedamzik, K., Fuller, G.M., and Mathews, G.J.: 1994, Astrophys. J. 423, 50 .

[22] Kainulainen, K., Kurki-Suonio, H. and Sihvola, E.: 1999, Phys. Rev. D 59, 083505 .

[23] Kang, H.-S. and Steigman, G.: 1992, Nucl. Phys. B 372, 494.

[24] Kneller, J.P., Scherrer, R.J., Steigman, G. and Walker, T.P.: 2001, astro$\mathrm{ph} / 0101386$.

[25] Kurki-Suonio, H., Matzner, R.A., Centrella, J.M., Rothman, T. and Wilson, J.: 1988, Phys. Rev. D 38, 1091.

[26] Kurki-Suonio, H. and Sihvola, E.: 2000, Phys. Rev. Lett. 84, 3756.

[27] Kurki-Suonio, H. and Sihvola, E.: 2000a, Phys. Rev. D 62, 103508.

[28] Kurki-Suonio, H. and Sihvola, E.: 2001, Phys. Rev. D 63, 083508.

[29] Lange, A.E. et al.: 2001, Phys. Rev. D 63, 042001.

[30] Leonard, R.E. and Scherrer, R.J.: 1996, Astrophys. J. 463, 420.

[31] Lesgourgues, J. and Liddle, A.R.: 2001, astro-ph/0105361.

[32] Levshakov, S.A., Dessauges-Zavadsky, M., D’Odorico, S. and Molaro. P.: 2001, astro-ph/0105529.

[33] Linsky, J.L.: 1998, Space Science Rev. 84, 285. 
[34] Lisi, E., Sarkar, S. and Villante, F.L.: 1999, Phys. Rev. D 59, 123520.

[35] Malaney, R.A. and Fowler, W.A.: 1988, Astrophys. J. 333, 14.

[36] Malaney, R.A. and Mathews, G.J.: 1993, Phys. Rep. 229, 145.

[37] Mathews, G.J., Meyer, B.S., Alcock, C.R. and Fuller, G.M.: 1990, Astrophys. J. 358, 36.

[38] Netterfield, C.B. et al.: 2001, astro-ph/0104460.

[39] O'Meara, J.M., Tytler, D, Kirkman, D., Suzuki, N., Prochaska, J.X., Lubin, D. and Wolfe, A.M.: 2001, Astrophys. J. 552, 718.

[40] Olive, K.A., Steigman, G. and Skillman, E.D.: 1997, Astrophys. J. 483, 788.

[41] Peimbert, M., Peimbert, A. and Ruiz, M.T.: 2000, astro-ph/0003154.

[42] Pettini, M. and Bowen D.V.: 2001, astro-ph/0104474.

[43] Pinsonneault, M.H., Walker, T.P., Steigman, G. and Narayanan, V.K.: 1999, Astrophys. J. 527, 180.

[44] Reeves, H.: 1991, Phys. Rep. 201, 335.

[45] Rehm, J.B. and Jedamzik, K.: 1998, Phys. Rev. Lett 81, 3307.

[46] Rehm, J.B. and Jedamzik, K.: 2001, Phys. Rev. D 63, 043509.

[47] Ryan, S.G., Norris, J.E. and Beers, T.C.: 1999, Astrophys. J. 523, 654.

[48] Ryan, S.G., Beers, T.C., Olive, K.A., Fields, B.D. and Norris, J.E.: 2000, Astrophys. J. 530, L57.

[49] Sihvola, E.: 2001, Phys. Rev. D 63, 103001.

[50] Sihvola, E.: 2001a, PhD thesis, Univ. of Helsinki.

[51] Smith, M.S., Kawano, L.H. and Malaney, R.A.: 1993, Astrophys. J. Suppl. Ser. 85, 219.

[52] Sonneborn, G., Tripp, T.M., Ferlet, R., Jenkins, E.B., Sofia, U.J., VidalMadjar, A. and Woźniak, P.R.: 2000, Astrophys. J. 545, 277.

[53] Stompor, R. et al.: 2001, astro-ph/0105062.

[54] Suzuki, T.K., Yoshii, Y. and Beers, T.C.: 2000, Astrophys. J. 540, 99.

[55] Thuan, T.X. and Izotov, Y.I.: 2001, this volume.

[56] Tytler, D., Burles, S., Lu, L., Fan, X.-M., Wolfe, A. and Savage, B.D.: 1999, Astron. J. 117, 63. 
[57] Vidal-Madjar, A.: 2001, astro-ph/0103170.

[58] Webb, J.K., Carswell, R.F., Lanzetta, K.M., Ferlet, R., Lemoine, M., VidalMadjar, A. and Bowen, D.V.: 1999, Nature 388, 250. 